\title{
ESTÍPITE DE PALMERA EN EL CAMPANIANO DEL GRUPO NEUQUÉN, PROVINCIA DEL NEUQUÉN, ARGENTINA
}

\author{
LEANDRO C. A. MARTÍNEZ ${ }^{1,2}$
}

'División Paleobotánica, Museo Argentino de Ciencias Naturales "Bernardino Rivadavia", Avda. Ángel Gallardo 470, C1405DJR, Buenos Aires, Argentina- Consejo Nacional de Investigaciones Científicas y Técnicas (CONICET). gesaghi@gmail.com

${ }^{2}$ División Paleobotánica, Facultad de Ciencias Naturales y Museo. Universidad Nacional de La Plata. Paseo del Bosque s/n, B1900FWA, La Plata, Argentina.

Resumen. En este trabajo se describe una nueva especie de palmera fósil procedente de la localidad de Auca Mahuevo, donde aflora la Formación Anacleto (Campaniano), en la provincia del Neuquén, Argentina. Los fósiles descriptos se corresponden a estípites de palmeras, asignados al género Palmoxylon Schenk. Los estípites fueron comparados con otras especies del mismo género y con representantes actuales de las Arecaceae, concluyéndose que pertenece a una nueva especie, denominada Palmoxylon garridoi sp. nov. Martínez. El hallazgo de palmeras en la Formación Anacleto permite sugerir la existencia de un clima cálido en el Campaniano del Grupo Neuquén, y además amplía el biocrón de este género en la Argentina.

Palabras clave. Cretácico. Arecaceae. Formación Anacleto. Cuenca Neuquina. Anatomía.

Abstract. PALM TRUNK IN THE CAMPANIAN OF NEUQUÉN GROUP, NEUQUÉN PROVINCE, ARGENTINA. In this paper a new species of fossil palm is described from the Auca Mahuevo locality, where the Anacleto Formation (Campanian) crops out, in the Neuquén province, Argentina. The described fossils correspond to palm trunks, referred to the genus Palmoxylon Schenk. These stems were compared with other species of this genus and with extant representatives of the family Arecaceae, and such comparisons allowed to assign it to a new species, Palmoxylon garridoi sp. nov. Martínez. The fossil record of palms in the Anacleto Formation suggests warm climate conditions in the Campanian of the Neuquén Group, and also extends the biochron of this genus in Argentina.

Key words. Cretaceous. Arecaceae. Anacleto Formation. Neuquén Basin. Anatomy.

LAs Arecaceae Berchtold y J. Presl, nom. cons. o Palmae Jussieu, nom. cons. et nom. alt. (palmeras) son un grupo exitoso de plantas que se distribuyen principalmente en regiones cálidas del planeta, compuesta por unos 190 géneros con más de 2000 especies vivientes, que conforman una parte muy importante y característica de la vegetación en los trópicos (Dransfield y Uhl, 1998; Simpson, 2006).

Las palmeras poseen una serie de adaptaciones únicas dentro de las monocotiledóneas, destacándose el crecimiento primario continuo en grosor de sus tallos, que junto con la lignificación gradual del parénquima y las fibras conllevan a que estas plantas adquieran un hábito arbóreo (Tomlinson, 2006).

Esta familia tiene un origen presumible en el Cretácico Temprano de acuerdo a estudios filogenéticos realizados sobre datos moleculares y también por las evidencias aportadas a través el registro fósil.

En base a las secuencias genómicas, se estima una edad comprendida entre los 128 y $115 \mathrm{Ma}$ para el stem group del orden Arecales (Magallón y Castillo, 2009). Por otra parte, las cifras obtenidas por otros investigadores datan al stem group de las Arecaceae en unos $120 \mathrm{Ma}$, divergiendo del crown group hace aproximadamente unos $110 \mathrm{Ma}$ (Janssen y Bremer, 2004).

Asumiendo que los granos de polen pertenecientes al género Monocolpopollenites H. D. Pflug y P. W. Thomson, del Albiano y Cenomaniano de África (Pan et al., 2006) se corresponden a Palmae, estos representarían a los registros fósiles más antiguos de dicha familia. Con respecto a los primeros restos megascópicos, los más confiables son impresiones de hojas denominadas Sabalites Saporta del Cretácico Tardío de América del Norte (Coniaciano-Santoniano temprano) y Austria (Campaniano temprano) (Harley, 2006). Además, se conocen rizomas, espinas y troncos fósiles de palmeras; los rizomas son ubicados en el morfogénero Rhizopalmoxylon Felix y las espinas en Spinophyllum Huard (Harley, 2006). Los estípites se agrupan en un único género: Palmoxylon Schenk, el cual contiene numerosos registros; entre los más antiguos están $P$. andegavense Crié y P. ligerinum Crié presumiblemente del Turoniano de Francia y P. cliffwoodensis Berry del Coniaciano-Santoniano de América del Norte (Harley, 2006).

En la Argentina, se conocen semillas y varios estípites de palmeras a partir del Maastrichtiano (Ancibor, 1995; 
Andreis et al., 1991), y desde entonces su registro ha sido continuo y abundante tanto en el Paleógeno (Arguijo, 1978, 1981; Romero, 1968; Petriella, 1972) como en el Neógeno (Lutz, 1980, 1984, 1986).

En el presente trabajo se describe una nueva especie del género Palmoxylon, proveniente de la Formación Anacleto (Campaniano), provincia del Neuquén; y constituye uno de los primeros estudios paleobotánicos realizados en dicha formación.

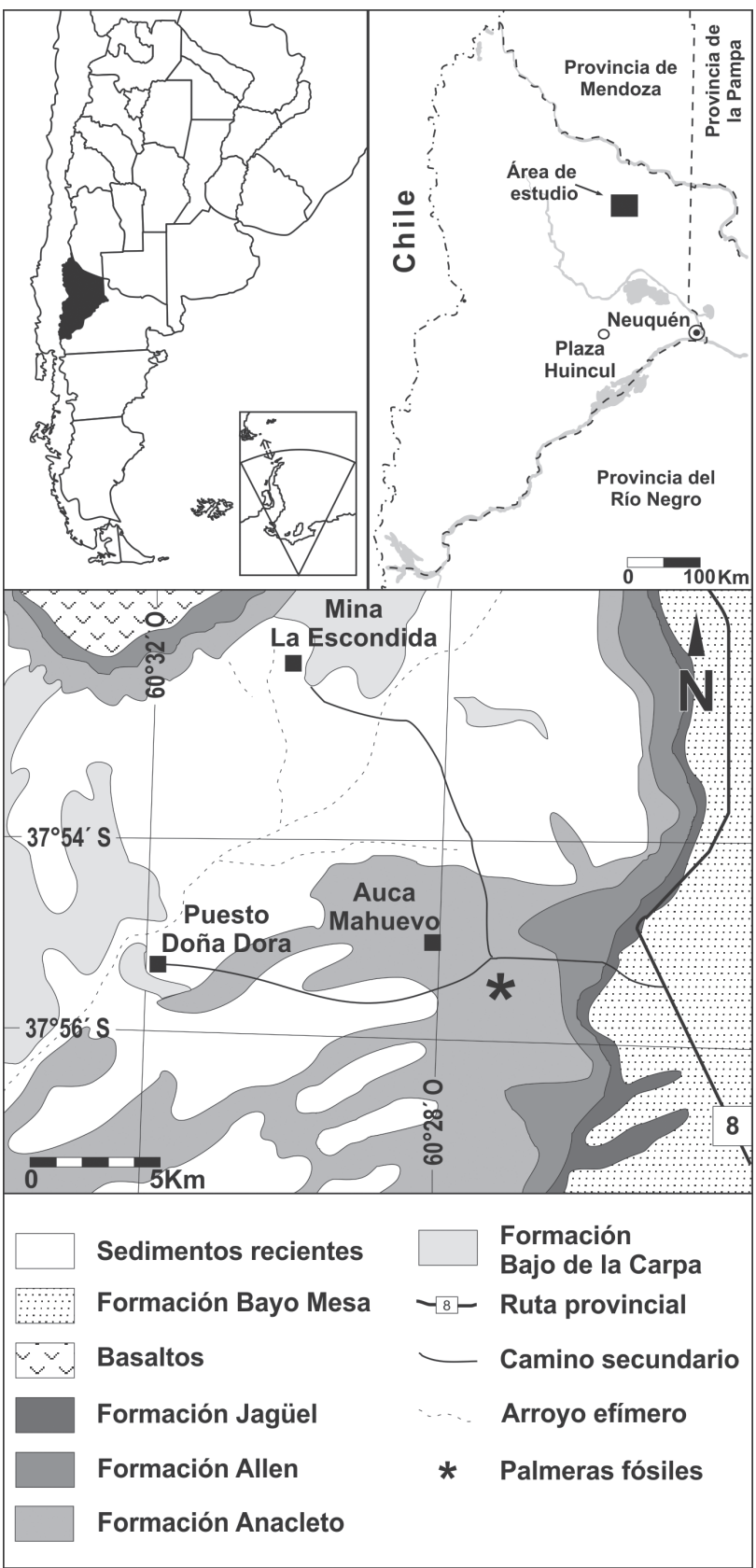

Figura 1. Mapa indicando la ubicación de la localidad fosilífera. Auca Mahuevo. Provincia del Neuquén, Argentina/ Map showing the fossiliferous locality. Auca Mahuevo. Neuquén province. Argentina.

\section{MARCO GEOGRÁFICO Y GEOLÓGICO}

Los materiales estudiados corresponden a 2 fragmentos de estípite silicificados, hallados del yacimiento Auca Mahuevo, en afloramientos correspondientes a la Formación Anacleto (Fig. 1) en el departamento de Ańelo, provincia del Neuquén, Argentina.

Herrero Ducloux (en Fossa Mancini et al., 1938) es quien define a los "Estratos de Anacleto", siendo Ramos (1981) quien la categoriza como Formación Anacleto. Es la unidad de mayor distribución del Grupo Neuquén (Subgrupo Río Colorado); su localidad tipo se ubica en el flanco sudoccidental del cerro Senillosa, cercano a la Aguada de Anacleto a unos $50 \mathrm{~km}$ al oeste de la ciudad de Neuquén (Garrido, 2000, 2010a; Leanza et al., 2004). La Formación Anacleto tiene una potencia que varía entre 85 a $100 \mathrm{~m}$, se apoya en concordancia sobre la Formación Bajo de la Carpa, y su techo se halla separado de las formaciones Allen y Loncoche por medio de una discordancia erosiva (Garrido, 2010a; Leanza et al., 2004; Legarreta y Gulisano, 1989).

Litológicamente, la Formación Anacleto (Fig. 2) está conformada por una sucesión de depósitos continentales compuestos por fangolitas rojizas a moradas, friables y macizas; entre los que se intercalan bancos de limolitas laminadas, de coloración gris-verdosa, junto a delgados paquetes de areniscas cuarzolíticas gris-amarillentas, con estratificación cruzada y marcas de ondulitas asimétricas (Garrido, 2000, 2010a).

La edad de la formación en Auca Mahuevo está determinada por estudios paleomagnéticos con una antigüedad comprendida entre los 83,5 a 79,5 Ma, es decir Campaniano (Dingus et al., 2000).

Las sedimentitas de la Formación Anacleto en Auca Mahuevo son depósitos finos de origen fluvial con presencia de caliche y paleosuelos de tipo vertisol, los que indicarían la ocurrencia de un paleoambiente con condiciones climáticas cálidas y fluctuantes (períodos húmedos y secos) (Garrido, 2010b).

Esta formación es mundialmente conocida por el hallazgo de nidadas de Megaloolithus Mikhailov con embriones de titanosaurios preservados en su interior; así como de otros restos de dinosaurios, tales como: Neuquensaurus australis Lydekker, Aucasaurus garridoi Coria, Chiappe y Dingus, y Gasparinisaura cincosaltensis Coria y Salgado (Chiappe et al., 1998, 2001, 2003; Coria et al., 2002; Leanza et al., 2004). Respecto a los registros paleobotánicos, recientemente se citó la presencia de restos permineralizados de gramíneas (Martínez, 2010 a, b). 


\section{MATERIALES Y MÉTODOS}

El estudio anatómico de los estípites fósiles se realizó por medio de cortes petrográficos, confeccionándose láminas delgadas en 2 sentidos para el estudio anatómico: corte transversal (CT) y corte longitudinal (CL). En las observaciones se utilizó un microscopio estereoscópico Leica Wild M3Z y un microscopio óptico (MO) Diaplan Leica; las fotografías se tomaron con las cámaras Nikon Coolpix L1 y Cannon Powershot S50.

En la descripción anatómica de los estípites se siguió a Tomlinson (1961) y la sistemática utilizada a nivel de subfamilias es la propuesta por Asmussen et al. (2006). Las medidas expuestas son el promedio de 25 mediciones y entre paréntesis figuran sus valores extremos (mínimo y máximo). Además se consultó la base de datos electrónica Palmweb (www.palmweb.org) para las descripciones, distribuciones y bibliografía de algunos taxones actuales.

Es de destacar que actualmente la monofilia de las Arecaceae está soportada por numerosos estudios que las agrupan dentro de las "Commelínidas" (sensu APG, 2009), contrario a algunas clasificaciones previas (e.g., Cronquist, 1981) las cuales asociaron a las Arecaceae dentro la subclase Arecidae Takhtajan; junto con las Araceae Jussieu, nom. cons. (Alismatales), Cyclanthaceae Richard, nom. cons. (Pandanales) y Pandanaceae Brown, nom. cons. (Pandanales) (APG, 1998; Givnish et al., 1999). En este trabajo la sistemática utilizada a nivel suprafamiliar se apoya en las bases previamente mencionadas.

Los materiales se encuentran depositados en la Colección del Museo Carmen Funes, (Plaza Huincul, Neuquén, Argentina) bajo el acrónimo PBPH.

\section{PALEONTOLOGÍA SISTEMÁTICA}

Familia Arecaceae C. H. Berchtold y J. Presl, nom. cons.

Género Palmoxylon Schenk 1882

Especie tipo. Palmoxylon blanfordii Schenk 1882.

\section{Palmoxylon garridoi}

Figuras 3,4 y 5

Derivación del nombre. La especie está dedicada al geólogo Alberto C. Garrido, por sus aportes al conocimiento sedimentológico y paleontológico de la Cuenca Neuquina, y en especial del Grupo Neuquén.

Diagnosis. Fragmento de estípite compuesto por parénquima fundamental y haces fibrovasculares dispersos al azar. Pa- rénquima fundamental formado por células isodiamétricas a amorfas, con espacios intercelulares asemejando un tejido lagunar. Estegmatas con cuerpos silíceos esferoidales, equinados y en series axiales. Parénquima tabular con 1 a 3 células de espesor rodeando a los haces fibrovasculares. Haces fibrovasculares sin orientación, con un pronunciado casquete de fibras reniformes a sub-circulares. Floema indiviso. Vasos protoxilemáticos numerosos y pequeños. Vasos metaxilemá-

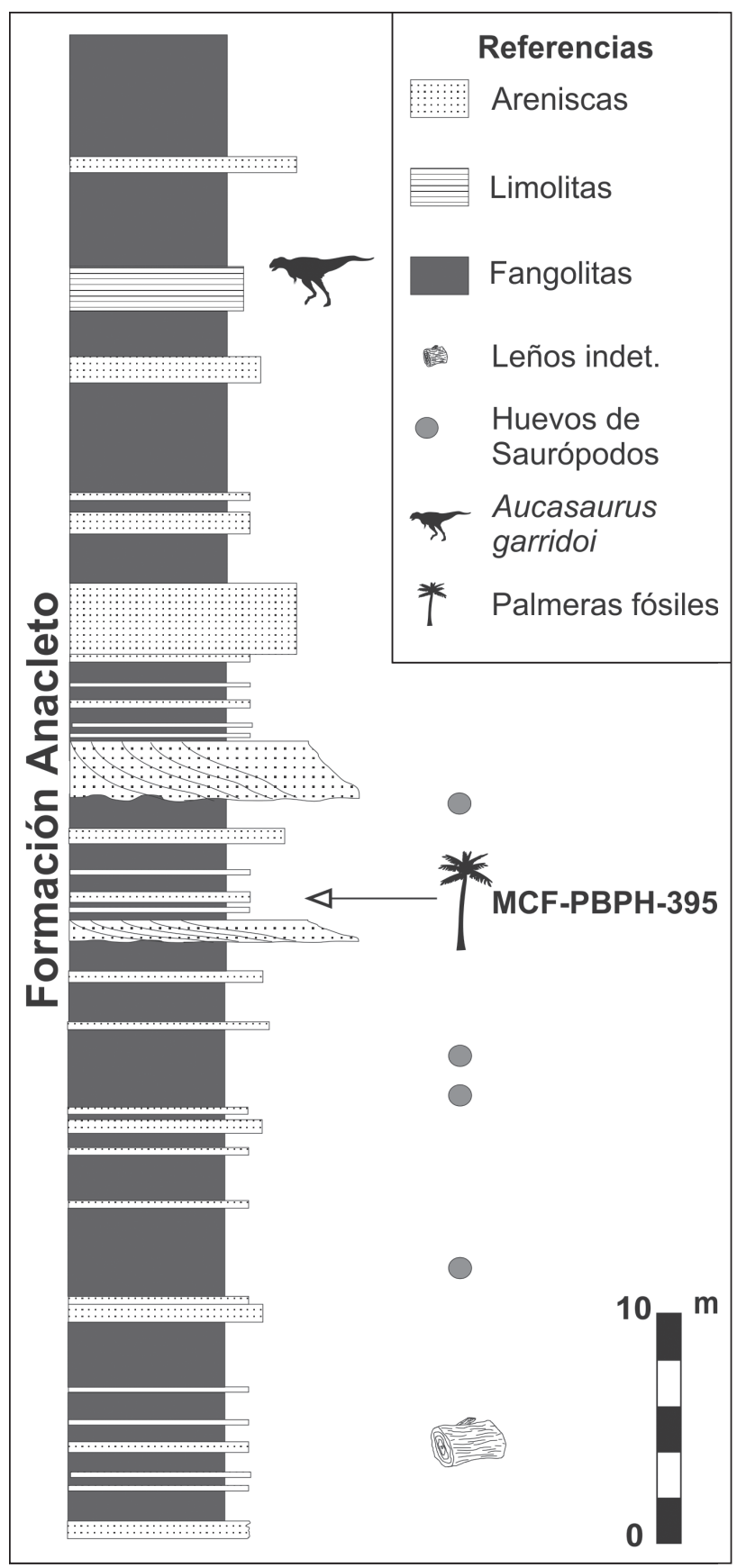

Figura 2. Perfil estratigráfico en la localidad fosilífera/ stratigraphic section in the fossiliferous locality. 
ticos en un número promedio de 3 (2-6) vasos por haz, con engrosamientos escalariformes, placas de perforación escalariformes o simples.

Diagnosis. Fragment of trunk composed by fundamental parenchyma cells and scattered fibrovascular bundles. Fundamental parenchyma consisting of isodiametric to amorphous cells, with intercellular spaces similar to a lacunar tissue. Stegmatas with spherical echinate silica-bodies in axial series. Tabular parenchyma 1 to 3 cells wide, sheathing the fibrovascular bundles. Fibrovascular bundles irregularly distributed, with a great reniform to subcircular sheath composed of sclerenchyma fibers. One phloem strand. Many and small protoxylem vessels. Metaxylem with a mean of 3 vessels (2-6) by bundle, with scalariform thickenings, and scalariform and simple perforation plates.

Holotipo. PBPH-395. PBPH-PMP-01. PBPH-PMP-02. PBPH-PMP-03.

Procedencia geográfica. Localidad "Auca Mahuevo". Auca Mahuida. Departamento de Añelo. Provincia del Neuquén, Argentina.

Procedencia estratigráfica. Formación Anacleto. Cretácico Superior (Campaniano).

Descripción. Los fósiles estudiados corresponden a dos fragmentos de estípites silicificados y decorticados. La descripción está basada principalmente en la pieza de mayor tamaño (PBPH-395), el cual posee $11 \mathrm{~cm}$ de ancho por $16 \mathrm{~cm} \mathrm{de}$ largo.

El fósil en corte transversal presenta haces fibrovasculares dispersos al azar. Los haces están inmersos en una matriz de células parenquimáticas y no presentan orientación alguna en sus elementos vasculares (Fig. 3.1-2).

Parénquima fundamental. En corte transversal se observa que está compuesto por células de paredes delgadas, isodiamétricas, alargadas, amorfas o de tamaño muy variable; entre las mismas se presentan espacios intercelulares o lagunas (Fig. 4.1-2). La mayoría de las células se hallan deformadas a causa del proceso de fosilización (Fig. 3.1-2). En corte longitudinal, las células del parénquima tampoco presentan ningún ordenamiento, en ciertos casos su tamaño y forma es muy variable, algunas de mayor longitud debido a un posterior desarrollo de un probable crecimiento secundario difuso (Fig. 3.4). Se observa la presencia de substancias obscuras en el interior de ciertas células, posiblemente representan taninos u otros depósitos (Fig. 3.5). Otras células, en cambio muestran contenidos esferoidales y hialinos atribuibles a gránulos de almidón, pudiéndose tratar de probables amiloplastos (Fig. 3.6, Fig. 5.5).
Estegmatas. Se disponen en series axiales, generalmente paralelos a las fibras, en su interior se hallan pequeños corpúsculos de sílice esferoidales y equinados (Fig. 3.7).

Haces fibrovasculares. Conformados por un parénquima tabular, un casquete de fibras, un haz de floema y un haz de xilema. En donde una vaina de parénquima tabular envuelve completamente a cada haz fibrovascular (Fig. 3.8-9). Los haces presentan un diámetro radial medio de 1266 (738-1551) $\mu \mathrm{m}$ y tangencial de $1000(503-1241) \mu \mathrm{m}$.

Parénquima tabular. En corte transversal, se compone de células poliédricas o radialmente alargadas, con paredes delgadas y en una disposición ordenada con 1 a 3 células de espesor que rodean a los haces fibrovasculares (Fig. 3.8-9, Fig. 5.1). En el corte longitudinal se observa que sus células son ordenadas, con sus paredes terminales en disposición horizontal (Fig. 5.2-4).

Fibras. En el corte transversal se observan fibras conformando un gran casquete generalmente reniforme a sub-circular (Fig. 3.8-9). En su cara interna limita con el floema y en la externa con el parénquima tabular (Fig. 3.8-9). Las fibras son elementos de paredes gruesas y lameladas, de contornos circular y con un diámetro medio de $49(22-71) \mu \mathrm{m}$. Axialmente, la longitud de las fibras no pudo ser medida, debido al estado de preservación (Fig. 5.2-3, 5).

Floema. Se halla ubicado entre el casquete de fibras y el xilema (Fig. 3.8-6). En la mayoría de los haces se observa que el floema está compuesto por 1 único paquete o cordón, de células pequeñas de contornos circulares y paredes delgadas. Xilema. Está compuesto por vasos protoxilemáticos y metaxilemáticos. En corte transversal el protoxilema se localiza en la región externa de los haces, limitando con el parénquima interfascicular y está compuesto por numerosos vasos de tamaño pequeño (Fig. 5.6). Axialmente se observa que sus paredes presentan engrosamientos anulares a helicoidales (Fig. 5.2-3). Debido a su mala preservación su longitud no pudo ser medida.

En el corte transversal el metaxilema se encuentra ubicado entre el protoxilema y el floema. Está conformado por vasos metaxilemáticos, con una media de 3 (2-6) vasos por haz (generalmente 2 vasos son los de mayor diámetro), un diámetro radial de $65(30-113) \mu \mathrm{m}$ y tangencial de 63 $(20-131) \mu \mathrm{m}$, de paredes delgadas con un diámetro de 3 (2-4) $\mu \mathrm{m}$ (Fig. 3.8-9, Fig. 5.6). Axialmente, presentan una longitud de 1214 (1016-1412) $\mu \mathrm{m}$ y muestran numerosos engrosamientos escalariformes (Fig. 5.2-3,7), con placas de perforación simples o escalariformes y de disposición oblicua (Fig. 5.8-9). En el interior de algunos vasos se observa la presencia de tilosis (Fig. 5.10). 

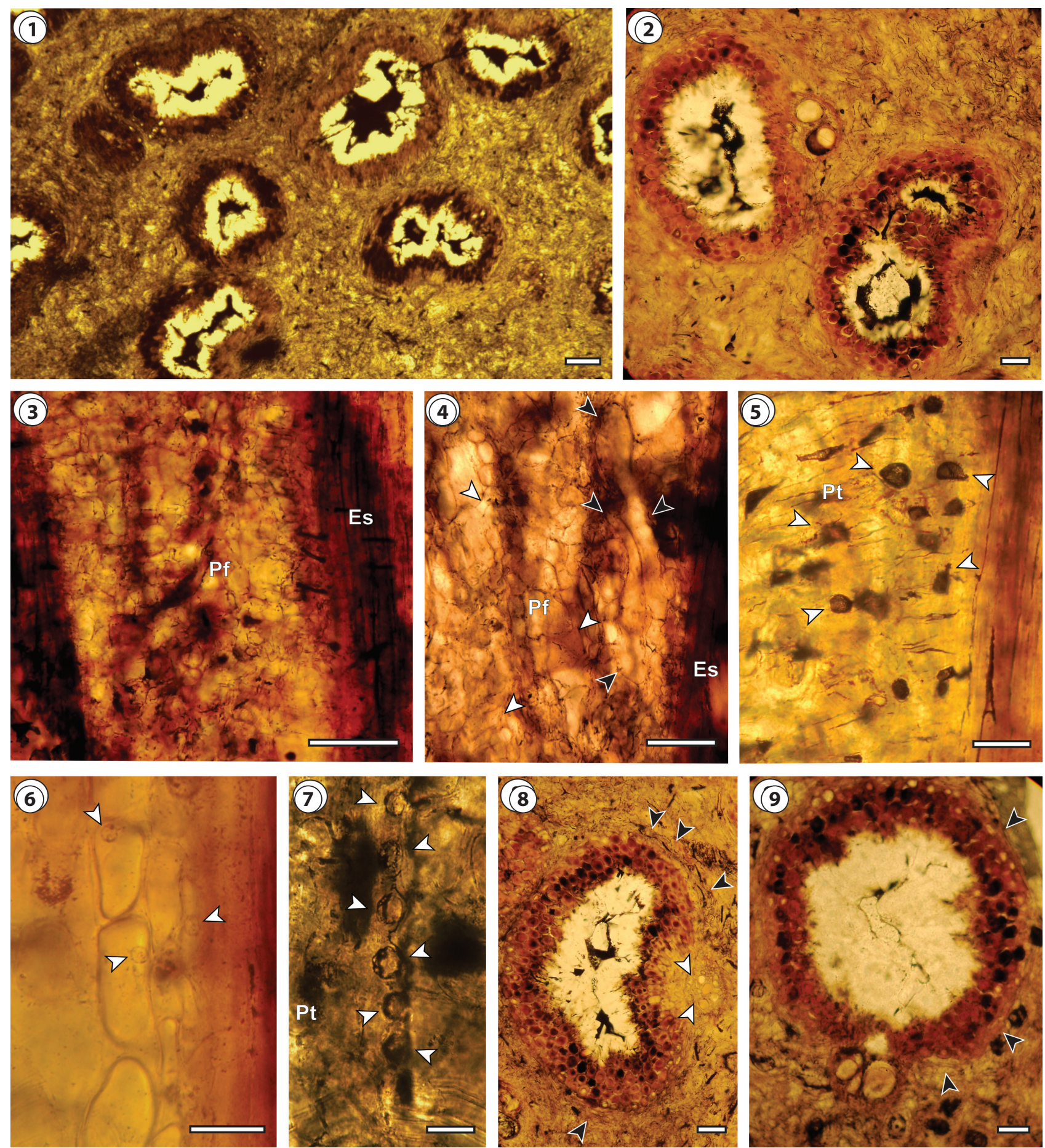

Figura 3. Palmoxylon garridoi sp. nov. Martínez (PBPH-395). 1-2, Corte transversal (CT)/ Cross section (CS); 1, aspecto general del tallo/general view of stem. Escala gráfica/ scale bar $=200 \mu \mathrm{m} ; \mathbf{2}$, haces fibrovasculares/ Fibrevascular bundles. Escala gráfica/ scale bar = 100 $\mu \mathrm{m}$. 3-7, corte longitudinal (CL)/ longitudinal section (LS); 3, parénquima fundamental/ fundamental parenchyma. Escala gráfica/ scale bar = 100 $\mu \mathrm{m} ; \mathbf{4}$, parénquima fundamental, con espacios intercelulares (flechas blancas) y células alargadas (¿crecimiento secundario difuso?) (flechas negras)/ fundamental parenchyma, with intercellular spaces (white arrows) and long cells (diffuse secondary growth?) (black arrows). Escala gráfica/ scale bar= $100 \mu \mathrm{m} ; \mathbf{5}$, células con posibles depósitos de tanino (flechas)/ cells with probable taniniferous deposits (arrows). Escala gráfica/ scale bar=100 $\mu$ m; $\mathbf{6}$, detalle de los amiloplastos conteniendo granos de almidón (flechas)/ detail of cells with starch (arrows). Escala gráfica/ scale bar = $25 \mu \mathrm{m} ; \mathbf{7}$, estegmatas con cuerpos de sílice (flechas)/ stegmatas with silica bodies (arrows). Escala gráfica/ scale bar $=25 \mu \mathrm{m} .8-9$, CT mostrando haces fibrovasculares. Se indican el floema (flechas blancas) y parénquima tabular (flechas negras)/ CT showing fibrovascular bundles. Phloem (white arrows) and tabular parenchyma (black arrows) are shown; 8, haz vascular con 6 vasos en el metaxilema/ vascular bundle with 6 metaxylem vessels. Escala gráfica/ scale bar $=100 \mu \mathrm{m}$; 9; haz vascular con 3 vasos metaxilemáticos/vascular bundle with 3 metaxylem vessels. Escala gráfica/scale bar $=100 \mu \mathrm{m}$. Pf. Parénquima fundamental/ground parenchyma. Pt. Parénquima tabular/ tabular parenchyma. Es. Fibras/ Fibres. Px. Protoxilema/ Protoxylem. Mx. Metaxilema/ Metaxylem. Fl. Floema / Phloem. 


\section{Comparaciones con estipites de Arecaceae actuales}

Tomlinson (1961), en su estudio sobre la anatomía de las palmeras, analizó los siguientes grupos: Arecoides, Bactroides, Borassoides, Caryotoides, Chamaedroides, Cocoides, Iriatoides, Lepidocaryoides, Sabaloides, Nypoides, Phoenicoides, Phytelephantoides, así como algunos géneros de afinidad incierta. Sin embargo, en la actualidad la mayoría de estos grupos fueron reagrupados o disueltos en base a estudios moleculares, y es así que Asmussen et al. (2006) sólo reconocen 5 Subfamilias: Calamoideae, Nypoideae, Coriphoideae, Ceroxyloideae y Arecoideae. En este estudio, las comparaciones con el material fósil aquí descripto siguen este último criterio, no obstante en tales comparaciones se incluyen entre paréntesis los grupos reconocidos por Tomlinson (1961; Tab. 1).

Los representantes de la subfamilia Calamoideae (Lepidocaryoides) son los más basales dentro de Palmae (Asmussen et al., 2006) y muchos de sus miembros poseen caracteres afines a Palmoxylon garridoi sp. nov. Entre ellos: Eremospatha (G. Mann y H. Wendland) H. Wendland, Calamus L., Myrialepis Beccari y Mauritia L. tienen haces vasculares con un masivo casquete de fibras, un sólo cordón de floema y 2 grandes vasos metaxilemáticos. En cambio otros géneros de esta subfamilia, como en: Ancistrophyllum (G. Mann y H. Wendland) H. Wendland, Daemonorops Blume, Korthalsia Blume y Plectocomia Martius ex J. A. Schultes y J. H. Schultes se distinguen de Palmoxylon garridoi sp. nov., porque presentan sólo 1 vaso metaxilemático y 2 bandas de floema; o un vaso y una banda de floema; o 2 bandas de floema y 2 vasos metaxilemáticos (Tomlinson, 1961).

Nypoideae (Nypoides) sólo cuenta con un único género,
Nypa Steck, que se distingue claramente tanto del fósil aquí estudiado como de todas las palmeras actuales debido a que los haces de fibras no están íntimamente asociados con los haces vasculares y por tener un importante desarrollo del parénquima aerífero, el cual le otorga al tallo una apariencia esponjosa (Kaul, 1981). Otra diferencia que presenta $N y p a$ es que posee estegmatas con fitolitos en "forma de sombrero" (Tomlinson, 1961).

En Coryphoideae (Caryotoides y Borasoides) existen géneros en los que se presenta un discreto paquete de fibras sólo sobre el floema, siendo además éste último indiviso, carácter que lo diferencia del casquete masivo que posee Palmoxylon garridoi. En otros géneros (Sabaloides) los haces siempre tienen el casquete de fibras bien constituido sobre el floema indiviso, sin embargo el metaxilema se distingue por tener vasos de gran tamańo en número variable según los géneros: 1 vaso (Licuala von Wurmb y Rhapis L.), 2 vasos (Erythea Watson, Livistona Brown y Serenoa Hooker) o numerosos vasos (Chamaerops L. y Trachycarpus Wendland). Los géneros que presentan 2 vasos poseen otros caracteres que lo distinguen de la especie fósil aquí estudiada. En Erythea las células parenquimáticas no presentan expansiones propias del crecimiento secundario difuso; Livistona usualmente posee el haz de floema subdividido; y en Serenoa las células del parénquima fundamental están tangencialmente extendidas, con haces vasculares difusos y vasos con placas de perforación escalariformes a reticuladas. Estos caracteres no son comunes al fósil de la Formación Anacleto.

De la subfamilia Ceroxyloideae (Ceroxylon Bonpland + Pseudophoenix H. Wendland ex Sargent + Phytelephantoides) sólo se cuenta con las descripciones del género Phytelephas Ruiz y Pavon, en el cual el casquete de fibras es abundante,
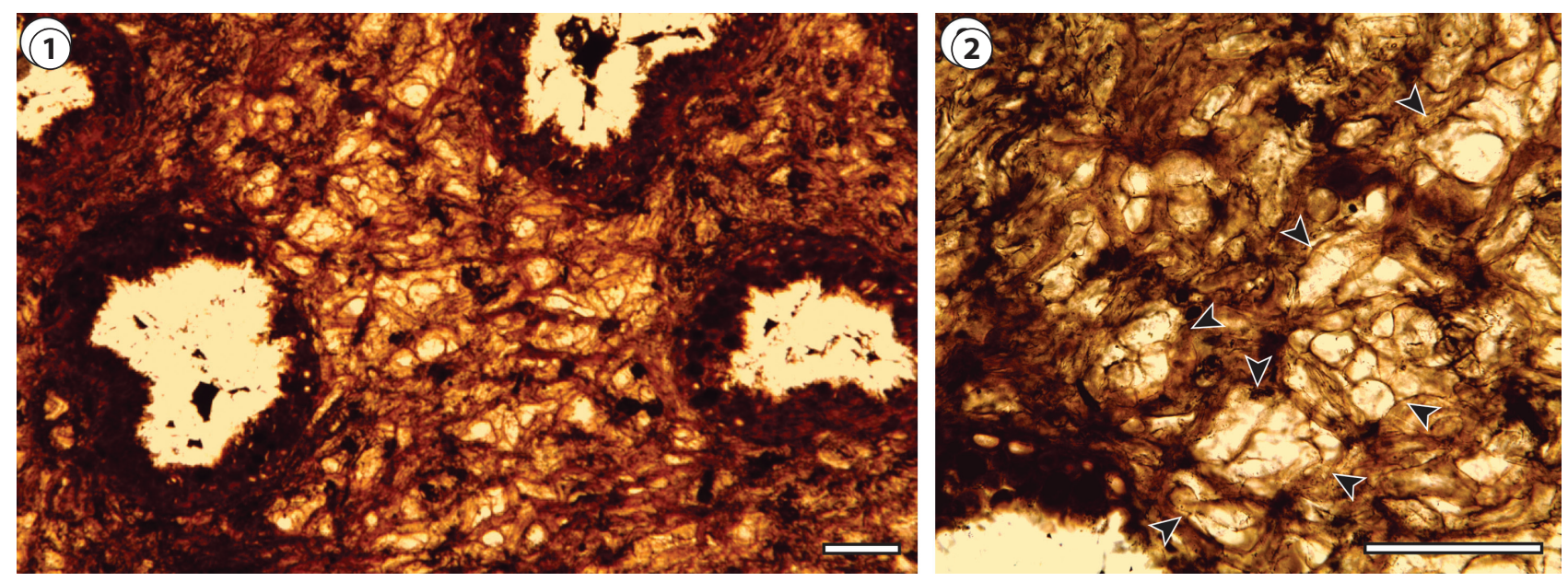

Figura 4. Palmoxylon garridoi sp. nov. Martínez (PBPH-395). 1-2. Corte transversal (CT)/ cross section (CS). 1. Parénquima fundamental con espacios intercelulares o lagunas/ fundamental parenchyma with intercellular spaces. Escala gráfica/ scale bar= $200 \mu \mathrm{m}$. 2. Detalle de las células del parénquima fundamental y espacios intercelulares (flechas)/ detail of fundamental parenchyma and intercellular spaces (arrows). Escala gráfica/ scale bar: $100 \mu \mathrm{m}$. 
le sigue el floema poco desarrollado e indiviso y por último el xilema con 2 o 3 vasos metaxilemáticos y un marcado desarrollo de parénquima con pequeños espacios intercelulares (Kaul, 1981). Esta serie de caracteres no es compatible con la especie fósil aquí descripta.

Finalmente, la subfamilia Arecoideae es la más diversificada y cuenta con numerosos géneros, muchos de los cuales aún no han sido descriptos, como es el caso de las Iriartoides sensu Tomlinson (1961). Entre los taxones estudiados, se hallan los que poseen 1 vaso metaxilemático (Arecoides) o 2 a más vasos metaxilemáticos (Chamaedoroideae), siendo no homologables con la descripción de Palmoxylon garridoi sp. nov. En cambio, Cocoides y Bactroides se asemejan a Palmoxylon garridoi sp. nov. por la presencia de 1 o 2 vasos metaxilemáticos, empero se diferencian del mismo por la escasa proporción de las fibras.

En base a las comparaciones realizadas se concluye que la nueva especie de Palmoxylon aquí descripta es afín a la subfamilia Calamoideae, pues comparten un gran desarrollo de los haces de fibras, número de vasos metaxilemáticos y disposición de un único haz de floema.

\section{Comparaciones con las especies del género Palmoxylon de América del Sur}

Entre los primeros trabajos detallados sobre palmeras fósiles se encuentra la monografía titulada Fossile Palmenhölzer realizada por Stenzel (1904). En ella se describe un total de 43 especies del género Palmoxylon, en donde el autor agru- pa a las especies del género en 5 tipos: (1) Tipo Maurita, (2) Tipo Corypha, (3) Tipo Kokos, (4) Radices Palmarum, y (5) Grupo incertae sedis. A su vez, cada uno de estos tipos presenta subdivisiones en diferentes grupos. Según está clasificación, Palmoxylon garridoi sp. nov. se corresponde con el Tipo Maurita en el Grupo Antiguensia.

Desde la creación del género Palmoxylon se han descripto numerosas especies en todo el mundo, existiendo una bibliografía muy extensa. Es por ello que en este trabajo las comparaciones se circunscriben a las especies sudamericanas (resumidas en la Tab. 2).

Palmoxylon garridoi sp. nov. posee un solo haz de floema en los haces fibrovasculares, carácter que lo diferencia de especies que presentan los haces de floema divisos, tales como: P. patagonicum Romero (1968), P. valchetense Ancibor (1995), P. santarosense Ancibor (1995) y P. rionegrense Ancibor (1995). Por otra parte, la nueva especie aquí descripta presenta estegmatas, en contraposición con $P$. pichaihuensis Ottone (2007) y P. concordiense Lutz $(1980,1986)$. En cuanto a $P$. bororoense Arguijo (1978) y P. varetum Arguijo (1981) difieren de la especie neuquina por tener un menor desarrollo en el haz de fibras. Por último, P. yuqueriense Lutz (1984) y P. chilensis Torres y Godoy (1982) se distinguen de Palmoxylon garridoi sp. nov. por presentar un menor número de vasos metaxilemáticos.

Cabe destacar que en la bibliografía existen especies de Palmoxylon que poseen espacios intercelulares, como es el caso de P. enochii Ruiz-Estrada y Cevallos-Ferriz (2009)

\begin{tabular}{|c|c|c|c|c|}
\hline $\begin{array}{l}\text { Subfamilias de Palmae } \\
\text { sensu Asmussen et al. } \\
\text { (2006) }\end{array}$ & $\begin{array}{l}\text { Grupo o taxón afín } \\
\text { sensu Tomlimson (1961) }\end{array}$ & $\begin{array}{l}N^{\circ} \text { promedio de vasos } \\
\text { en el metaxilema }\end{array}$ & Floema & Fibras \\
\hline Calamoideae & Lepidocaryoides & 1 ó 2 & En 2 bandas o indiviso & Muy desarrolladas \\
\hline Nypoideae & Nypoides & $1 a 3$ & indiviso & Poco desarrolladas \\
\hline \multirow[t]{4}{*}{ Coryphoideae } & Caryotoides & 2 & Indiviso & Poco desarrolladas \\
\hline & Borasoides & 1 ó 2 & Indiviso & Desarrolladas \\
\hline & Sabaloides & 1 ó 2 & Indiviso & Muy desarrolladas \\
\hline & Phoenicoides & 2 & Indiviso & Muy desarrolladas \\
\hline Ceroxyloideae & $\begin{array}{l}\text { Ceroxylon, Pseudophoenix y } \\
\text { Phytelephantoides }\end{array}$ & 2 ó 3 & Indiviso & Muy desarrolladas \\
\hline \multirow[t]{5}{*}{ Arecoideae } & Arecoides & 1 & Indiviso & Poco desarrolladas \\
\hline & Cocoides & $\begin{array}{l}1 \text { en Elaeis } N . J . \text { Jac- } \\
\text { quin y } 2 \text { en } \operatorname{Cocos} L \text {. }\end{array}$ & Indiviso & Poco desarrolladas \\
\hline & Bactroides & 2 & Indiviso & Muy poco desarrolladas \\
\hline & Chamaedoroides & 2 omás & Indiviso & Poco desarrolladas \\
\hline & Iriatoides & ¿? & ¿? & ¿? \\
\hline
\end{tabular}



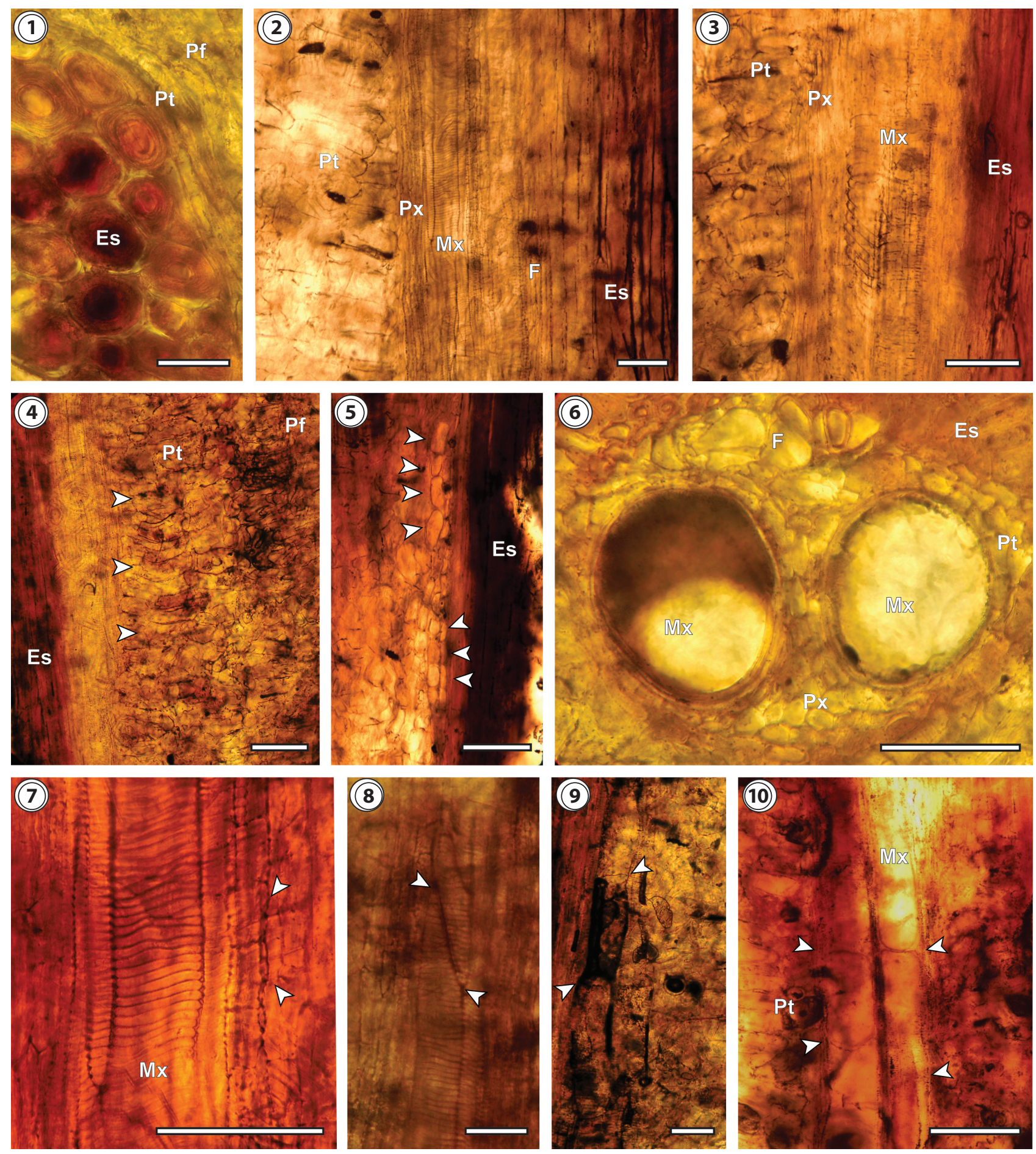

Figura 5. Palmoxylon garridoi sp. nov. Martínez (PBPH-395). 1. Corte transversal (CT). Fibras y células del parénquima tabular/ cross section (CT).

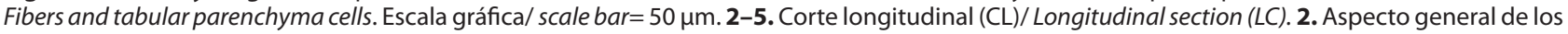
haces fibrovasculares/ General view of fibrevascular bundles. Escala gráfica/ scale bar $=100 \mu \mathrm{m}$. 3. Detalle de los haces fibrovasculares/ Fibrevascular bundles, detail. Escala gráfica/scale bar $=100 \mu \mathrm{m}$. 4. Detalle de las fibras y del parénquima tabular (flechas)/ fibres and tabular parenchyma, detail (arrows). Escala gráfica/ scale bar $=50$ um. 5. Células del parénquima (¿amiloplastos? flechas)/ parenchyma cells (with starch grains? arrows). Escala gráfica/ scale bar $=50 \mu \mathrm{m}$. 6. CT. Detalle del haz vascular/ Cross section. Vascular bundle, detail. Escala gráfica/ scale bar=100 $\mu \mathrm{m}$. 7-10. CL/ LC. 7. Detalle de miembro de vaso con engrosamientos y parénquima (flechas)/ detail of vessel with thickenings and parenchyma (arrows). Escala gráfica/ Scale bar $=100 \mu \mathrm{m}$. 8. Placa de perforación escalariforme (flecha)/ scalariform perforation plate (arrows). Escala gráfica/ scale bar: $100 \mu \mathrm{m}$. 9. Placa de perforación simple (flecha)/ simple perforation plate (arrow). Escala gráfica/ scale bar $=100 \mu \mathrm{m}$. 10. Vasos con tílides (flechas)/ vessels with tyloses (arrows). Escala gráfica/ scale bar $=100 \mu \mathrm{m}$. Pf. Parénquima fundamental/ ground parenchyma. Pt. Parénquima tabular/ tabular parenchyma. Es. Fibras/ Fibres. Px. Protoxilema/ Protoxylem. Mx. Metaxilema/ Metaxylem. Fl. Floema/ Phloem. 


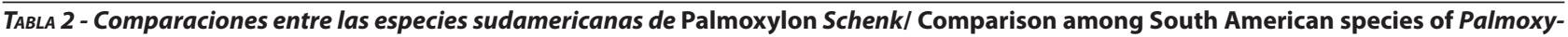
lon Schenk

\begin{tabular}{|c|c|c|c|c|c|c|c|c|c|}
\hline & $\begin{array}{l}\text { Edady } \\
\text { procedencia }\end{array}$ & $\begin{array}{l}\text { Sección del } \\
\text { estípite }\end{array}$ & $\begin{array}{l}\text { Forma del } \\
\text { haz de fibras } \\
\text { sensu Stenzel }\end{array}$ & $\begin{array}{l}\text { Desarrollo } \\
\text { del haz de } \\
\text { fibras }\end{array}$ & FI. & Mx. & Vs. & $P q$. & Es. \\
\hline $\begin{array}{l}\text { Palmoxylon } \\
\text { garridoi sp. } \\
\text { nov. Martínez }\end{array}$ & $\begin{array}{l}\text { Cretácico } \\
\text { Neuquén, } \\
\text { Argentina }\end{array}$ & $\begin{array}{l}\text { Fragmento } \\
\text { del estípite }\end{array}$ & Antiguensia & $m d$. & $1 \mathrm{haz}$ & $\begin{array}{l}3 \text { vasos } \\
(2-6)\end{array}$ & $\begin{array}{l}\text { Engrosamientos } \\
\text { anulares, helicoida- } \\
\text { les y escalariformes. } \\
\text { Placas escalari- } \\
\text { formes y simples }\end{array}$ & $\begin{array}{l}\text { pf. } y \\
\text { pr. }\end{array}$ & $p$ \\
\hline $\begin{array}{l}\text { Palmoxylon } \\
\text { santarosense } \\
\text { Ancibor (1995) }\end{array}$ & $\begin{array}{l}\text { Cretácico } \\
\text { Río Negro, } \\
\text { Argentina }\end{array}$ & Estípite & Reniforme & $m d$. & $\begin{array}{l}1 \text { haz } \\
\text { lobulado, } \\
\text { a veces } 2 \\
\text { haces }\end{array}$ & $\begin{array}{l}1 \text { ó } 2 \\
\text { vasos }\end{array}$ & $\begin{array}{l}\text { Engrosamientos } \\
\text { helicoidales. } \\
\text { Placas escalari- } \\
\text { formes }\end{array}$ & $p f$ & $p$ \\
\hline $\begin{array}{l}\text { Palmoxylon } \\
\text { rionegrense } \\
\text { Ancibor (1995) }\end{array}$ & $\begin{array}{l}\text { Cretácico } \\
\text { Río Negro, } \\
\text { Argentina }\end{array}$ & Estípite & $\begin{array}{l}\text { Lunariay } \\
\text { reniforme }\end{array}$ & $d$ & $\begin{array}{l}\text { Lobulado } \\
\text { y sepa- } \\
\text { rado hasta } \\
\text { en } 4 \text { haces }\end{array}$ & 4-6 vasos & $\begin{array}{l}\text { Engrosamientos } \\
\text { anulares y helicoi- } \\
\text { dales. Placas esca- } \\
\text { lariformes }\end{array}$ & $i ?$ & a. \\
\hline $\begin{array}{l}\text { Palmoxylon } \\
\text { valchetense } \\
\text { Ancibor (1995) }\end{array}$ & $\begin{array}{l}\text { Cretácico } \\
\text { Río Negro, } \\
\text { Argentina }\end{array}$ & Estípite & $\begin{array}{l}\text { Lunariay } \\
\text { reniforme }\end{array}$ & $m d$. & $\begin{array}{l}2 \text { haces } \\
\text { o muy } \\
\text { lobulado }\end{array}$ & $\begin{array}{l}2 \text { a } 3 \text { va- } \\
\text { sos, raras } \\
\text { veces } 4 \\
\text { vasos }\end{array}$ & $\begin{array}{l}\text { Placas } \\
\text { escalariformes }\end{array}$ & pf. & a. \\
\hline $\begin{array}{l}\text { Palmoxylon } \\
\text { chilensis Torres } \\
\text { y Godoy (1982) }\end{array}$ & $\begin{array}{l}\text { ¿Cretácico } \\
\text { Superior? } \\
\text { Chile }\end{array}$ & Estípite & Complanata & $d$ & 1 haz & 1 vaso & $\begin{array}{l}\text { Placas } \\
\text { escalariformes }\end{array}$ & pf. ypr. & $a$. \\
\hline $\begin{array}{l}\text { Palmoxylon } \\
\text { pichaihuensis } \\
\text { Ottone (2007) }\end{array}$ & $\begin{array}{l}\text { Cretácico - } \\
\text { Paleógeno } \\
\text { Neuquén, } \\
\text { Argentina }\end{array}$ & Estípite & Reniforme & $d$ & ¿? & 1-5 vasos & $\begin{array}{l}\text { Engrosamientos } \\
\text { anularesy } \\
\text { helicoidales }\end{array}$ & ¿? & a. \\
\hline $\begin{array}{l}\text { Palmoxylon } \\
\text { patagonicum } \\
\text { Romero (1968) }\end{array}$ & $\begin{array}{l}\text { Paleógeno } \\
\text { Chubut, } \\
\text { Argentina }\end{array}$ & Estípite & $\begin{array}{l}\text { Complanatay } \\
\text { cordata }\end{array}$ & $d$ & 1-2 haces & $\begin{array}{l}2(1-4) \\
\text { vasos }\end{array}$ & $\begin{array}{l}\text { Engrosamientos } \\
\text { anulares, helicoida- } \\
\text { les y escalariformes. } \\
\text { Placas escalari- } \\
\text { formes }\end{array}$ & pf. ypr. & p. \\
\hline $\begin{array}{l}\text { Palmoxylon } \\
\text { bororense } \\
\text { Arguijo (1979) }\end{array}$ & $\begin{array}{l}\text { Paleógeno } \\
\text { Chubut, } \\
\text { Argentina }\end{array}$ & $\begin{array}{l}\text { Cilindro cen- } \\
\text { tral y área } \\
\text { subdermal }\end{array}$ & $\begin{array}{l}\text { Vaginatay } \\
\text { reniforme }\end{array}$ & $d$ & ¿? & 2-4 vasos & $\begin{array}{l}\text { Engrosamientos } \\
\text { escalariformes. } \\
\text { Placas escalari- } \\
\text { formes }\end{array}$ & pf. & $p$ \\
\hline $\begin{array}{l}\text { Palmoxylon } \\
\text { varetum } \\
\text { Arguijo (1981) }\end{array}$ & $\begin{array}{l}\text { Paleógeno } \\
\text { Chubut, } \\
\text { Argentina }\end{array}$ & $\begin{array}{l}\text { Centro del } \\
\text { estípite }\end{array}$ & Vaginata & $d$ & $1 \mathrm{haz}$ & 27 vasos & $\begin{array}{l}\text { Engrosamientos } \\
\text { escalariformes. } \\
\text { Placas escalari- } \\
\text { formes }\end{array}$ & pf.ypr. & $p$ \\
\hline $\begin{array}{l}\text { Palmoxylon } \\
\text { concordiense } \\
\text { Lutz (1980, } \\
\text { 1986) }\end{array}$ & $\begin{array}{l}\text { Plioceno } \\
\text { Entre Ríos, } \\
\text { Argentina }\end{array}$ & $\begin{array}{l}\text { Parte basal } \\
\text { yestípite }\end{array}$ & $\begin{array}{l}\text { Sagittata, } \\
\text { complanatay } \\
\text { cordata }\end{array}$ & $m d$. & $1 \mathrm{haz}$ & 2 vasos & $\begin{array}{l}\text { Engrosamientos } \\
\text { escalariformes }\end{array}$ & $\begin{array}{l}\text { pf.., pr. } \\
\text { ypt. }\end{array}$ & $a$. \\
\hline $\begin{array}{l}\text { Palmoxylon } \\
\text { yuqueriense } \\
\text { Lutz (1984) }\end{array}$ & $\begin{array}{l}\text { Plioceno } \\
\text { Entre Ríos, } \\
\text { Argentina }\end{array}$ & Estípite & $\begin{array}{l}\text { Complanata, } \\
\text { cordatay } \\
\text { lunaria }\end{array}$ & $m d$. & $1 \mathrm{haz}$ & 2 vasos & $\begin{array}{l}\text { Engrosamientos } \\
\text { escalariformes }\end{array}$ & $\begin{array}{l}\text { pf., pt. } \\
\text { ypt. }\end{array}$ & $p$ \\
\hline
\end{tabular}

Fl. Floema. Mx. Metaxilema. Vs. Vasos. Pq. Parénquima. Es. Estegmatas. md. muy desarrollado. d. desarrollado. p. presentes. a. ausentes. pf. Parénquima fundamental. pr. Parénquima radial. pt. Parénquima tabular. 
del Cretácico Superior de México. Sin embargo, se diferencia anatómicamente de $P$. garridoi porque tienen un mayor desarrollo de los espacios intercelulares, como así también por la presencia vasos con de placas de perforación simples y punteaduras opuestas en la especie mexicana.

Por medio de las comparaciones aquí expuestas se establece que Palmoxylon garridoi sp. nov. es una nueva especie de palmera afín a la subfamilia Calamoideae claramente distinguible del resto de los estípites de palmeras fósiles.

\section{RECONSTRUCCIÓN PALEOAMBIENTAL}

En la localidad Auca Mahuevo, además de los fragmentos de estípite de Palmoxylon garridoi sp. nov., se hallaron numerosos tallos o cańas permineralizadas afines a la familia Gramineae Juss. nom. alt. et cons. o Poaceae Barnhart nom. cons. (gramíneas) junto con restos indeterminados de tallos de dicotiledóneas herbáceas y troncos de coníferas (Martínez 2010a, b).

Tomando en su conjunto a los datos aportados, tanto por los restos paleobotánicos como por la sedimentología, es posible sugerir una reconstrucción ambiental para la Formación Anacleto en la localidad Auca Mahuevo. La información brindada por la geología, indica que los sedimentos en el área de estudio se corresponden a depósitos finos de origen fluvial; mencionándose además la presencia de caliche y paleosuelos de tipo vertisol, los que señalarían la ocurrencia de un paleoambiente con condiciones climáticas cálidas y fluctuantes (períodos húmedos y secos). Es de destacar que los niveles fosilíferos expuestos muestran una mayor concentración de las plantas como de Megaloolithus, tanto en los depósitos de paleosuelos como en los canales abandonados, indicando que estos últimos habrían prosperado entre antiguas áreas de albardón y fajas de meandros, a la vera de los cursos de agua (Garrido, 2010b).

Las probables condiciones ecológicas en las que habitaba la flora exhumada en Auca Mahuevo fueron inferidas considerando el ambiente en el que se viven sus representantes actuales. En sendos casos, las Arecaceae (Calamoideae) y Gramineae son de distribución principalmente tropical (Cronquist, 1981; Dransfield y Uhl, 1998; Simpson, 2006). Desde este punto de vista, las palmeras son indicadoras paleoclimáticas muy precisas, ya que a partir de su aparición en el Cretácico la expansión y retracción de las palmeras a lo largo del tiempo ha sido concomitante con las áreas tropicales; es por ello que su registro señala condiciones cálidas (Harley, 2006). Por su parte, en el presente las Gramineae (si bien son cosmopolitas) tienen mayor de- sarrollo en áreas tropicales, como asimismo en ambientes áridos o semiáridos del planeta (Cronquist, 1981). Algunas Arecaceae, como la mayoría de las Poaceae, presentan polinización anemófila (Henderson, 1986; Gibson, 2009) que caracteriza generalmente a las plantas que viven en lugares abiertos como lo son las sabanas o praderas, lo que posiblemente estaría indicando que Auca Mahuevo sería una zona abierta con vegetación herbácea dominante y pocos elementos de porte arbóreo.

\section{CONCLUSIONES}

Dentro de Arecaceae, la subfamilia Calamoideae ocupa una posición basal y en la actualidad no se cuenta con representantes en Argentina. Es por ello que la presencia de restos asignables a esta subfamilia (en este caso, Palmoxylon garridoi sp. nov.) representa el registro más austral para las Calamoideae, indicando además condiciones tropicales durante el depósito de la Formación Anacleto.

El hallazgo de Palmoxylon garridoi sp. nov. se condice con el modelo sedimentario propuesto por Garrido (2010b), el cual sugiere que el paleoclima reinante en el momento de acumulación de los sedimentos que originaron a la Formación Anacleto, y en especial en Auca Mahuevo, estaría caracterizado por condiciones cálidas con periodos alternantes de sequía y humedad. En este contexto las comunidades de Gramineae y Palmae habrían crecido sobre una planicie de inundación sometida ocasionalmente a pulsos de inundación y sequía (Martínez, 2010 a, b). Este proceso habría otorgado un entorno óptimo que permite proponer la probable existencia de ambientes abiertos en los que habría crecido una abundante flora herbácea, que estaría constituida fundamentalmente por Poaceae junto a una vegetación arbórea representada por Arecaceae (Palmoxylon garridoi sp. nov.).

El registro en Auca Mahuevo de Poaceae y Palmae, establecería la posible presencia de una flora compuesta principalmente de Magnoliophytas (gramíneas y palmeras) ya en el Campaniano temprano de la Cuenca Neuquina; conformando un ambiente de tipo sabana, muy eventualmente asociada con cursos de agua, bajo condiciones climáticas tropicales a subtropicales.

Finalmente, cabe destacar que las comunidades dominadas por monocotiledóneas (gramíneas y palmeras), no se conocían en el Campaniano del planeta, como tampoco su vinculación tan estrecha con los sitios de nidificación de dinosaurios. 


\section{AGRADECIMIENTOS}

Este trabajo fue desarrollado bajo el marco de la Tesis "Estudios Xilológicos en el Cretácico de la Cuenca Neuquina, provincia del Neuquén, Argentina” financiado parcialmente por las Becas Doctorales Tipo I y Tipo II otorgadas al autor por el Consejo Nacional de Investigaciones Científicas y Técnicas (CONICET).

Este trabajo también fue desarrollado bajo los proyectos CONICET PIP 5093 y PIP 679 bajo la dirección de G. del Fueyo. Finalmente quiero agradecer los árbitros G. Ottone, y E. Ruiz-Estrada, y a los editores Mariana Brea y Ezequiel Vera quienes por medio de sus correcciones y sugerencias ayudaron a mejorar este trabajo.

\section{BIBLIOGRAFÍA}

Ancibor, E. 1995. Palmeras fósiles del Cretácico Tardío de la Patagonia Argentina (Bajo de Santa Rosa, Río Negro). Ameghiniana 32: 287-299.

Andreis, R.R., Ancibor, E., Archangelsky, S., Artabe, A., Bonaparte, J. y Genise, J. 1991. Asociación de vegetales y animales en estratos del Cretácico Tardío del norte de Patagonia. Ameghiniana 28: 201-202.

APG. 1998. An ordinal classification for the families of flowering plants. Annals of the Missouri Botanical Garden 85: 531-553.

APG. 2009. An update of the Angiosperm Phylogeny Group classification for the orders and families of flowering plants: APG III. Botanical Journal of the Linnean Society 161: 106-121.

Arguijo, M.H. 1978. Palmoxylon bororoense n. sp. de la Formación Cerro Bororó (Paleoceno), provincia de Chubut, Argentina. Physis 38: 87-96.

Arguijo, M.H. 1981. Palmoxylon vaterum, n. sp. del Paleoceno (Daniano) de la provincia de Chubut, Argentina. Physis 39: 49-59.

Asmussen, C.A., Dransfield, J., Deickmann, V., Barford, A.S., Pintaud, J.C. y Baker, W.J. 2006. A new subfamily classification of the palm family (Arecaceae): evidence from plastid DNA phylogeny. Botanical Journal of the Linnean Society 151: 15-38.

Chiappe, L.M., Coria, R.A., Dingus, L., Jackson, F., Chinsamy, A. y Fox, M. 1998. Sauropod dinosaur embryos from the Late Cretaceous of Patagonia. Nature 396: 258-261.

Chiappe, L.M., Coria, R.A., Jackson, F. y Dingus, L. 2003. The Late Cretaceous nesting site of Auca Mahuevo (Patagonia, Argentina): Eggs, nests, and embryos of titanosaurian sauropods. Palaeovertebrata 43: 97-108.

Chiappe, L.M., Salgado, L. y Coria, R.A. 2001. Embryonic skulls of titanosaur sauropod dinosaurs. Science 293: 2444-2446.

Coria, R.A., Chiappe, L.M. y Dingus, L. 2002. A new close relative of Carnotaurus sastrei Bonaparte, 1985 (Theropoda: Abelisauridae) from the Late Cretaceous of Patagonia. Journal of Vertebrate Palaentology 22: $460-465$.

Cronquist, A. 1981. An Integrated System of Classification of Flowering Plants. Columbia Univ. Press. New York, 1262 p.

Dingus, L., Clarke, J., Scott, G.R., Swisher III, C.C., Chiappe, L.M. y Coria, R.A. 2000. Stratigraphy and magnetostratigraphic/faunal constraints for the age of sauropod embryo-bearing rocks in the Neuquén Group (Late Cretaceous, Neuquén province, Argentina). American Museum Novitates 3290: 1-11.

Dransfield, J. y Uhl, N.W. 1998. Palmae. En: K. Kubitzki (Ed.). The Families and Genera of Vascular Plants. IV. Flowering Plants. Monocotyledons. Alismatanae and Commelinanae (except Gramineae). Springer, Berlin, p. 306-388.

Fossa-Mancini, E., Feruglio, E. y Yussen de Campana, J.C. 1938. Una reunión de geólogos de Y.P.F. y el problema de la terminología estratigráfica. Boletín de Informaciones Petroleras 15: 1-67.

Garrido, A.C. 2000. [Estudio estratigráfico y reconstrucción paleoambiental de las secuencias fosiliferas continentales del Cretácico Superior en las inme- diaciones de Plaza Huincul, provincia del Neuquén. Trabajo Final para el Título de Grado en Ciencias Geológicas. Facultad de Ciencias Exactas, Físicas y Naturales, Universidad Nacional de Córdoba, Córdoba, Argentina, 78 p. Inédito.].

Garrido, A. 2010a. Estratigrafía del Grupo Neuquén, Cretácico Superior de la Cuenca Neuquina (Argentina): nueva propuesta de ordenamiento litoestratigráfico. Revista del Museo Argentino de Ciencias Naturales n. s. 12: $121-177$

Garrido, A. 2010b. Paleoenvironment of the Auca Mahuevo and Los Barreales sauropod nesting-sites (Late Cretaceous, Neuquén Province, Argentina). Ameghiniana 47: 99-106.

Gibson, D.J. 2009. Grasses and Grassland Ecology. Oxford Universiry Press. New York, 305 p.

Givnish, T.J., Evans, T.M., Pires, J.C. y Sytsma, K.J. 1999. Polyphyly and convergent morphological evolution in Commelinales and Commelinidae: evidence from rbcL sequence data. Molecular Phylogenetics and Evolution 12: 360-385.

Harley, M.M. 2006. A summary of fossil records for Arecaceae. Botanical Journal of the Linnean Society 151: 39-67.

Henderson, A. 1986. A review of pollination studies in the Palmae. The Botanical Review 52: 221-259.

Janssen, T. y Bremer, K. 2004. The age of major monocot groups inferred from $800+r b c L$ sequences. Botanical Journal of the Linnean Society 146: 385-398.

Kaul, KN. 1981. Anatomy of palm stems - II. The Palaeobotanist 28.29: 447-454.

Leanza, H.A., Apesteguía, S., Novas, F.E. y de la Fuente, M.S. 2004. Cretaceous terrestrial beds from the Neuquén Basin (Argentina) and their tetrapod assemblages. Cretaceous Research 25: 61-87.

Legarreta, L. y Gulisano, C. A. 1989. Análisis estratigráfico secuencial de la Cuenca Neuquina. (Triásico superior - Terciario inferior). En: G.A. Cheloli y L.A. Spalletti; (Eds.), Cuencas Sedimentarias Argentinas, Serie Correlación Geológica 6: 221-243.

Lutz, A.I. 1980. Palmoxylon concordiense n. sp. del Plioceno de la provincia de Entre Ríos, República Argentina. $2^{\circ}$ Congreso Argentino de Paleontologia y Bioestratigrafía y $1^{\circ}$ Congreso Latinoamericano de Paleontología, Actas 3: $129-140$

Lutz, A.I. 1984. Palmoxylon yuqueriense n. sp. del Plioceno de la Provincia de Entre Ríos, Argentina. $3^{\circ}$ Congreso Argentino de Paleontología y Bioestratigrafia (Corrientes), Actas: 197-207.

Lutz, A.I. 1986. Descripción morfo-anatómica del estípite de Palmoxylon concordiense Lutz del Plioceno de Entre Ríos, Argentina. FACENA 6: 17-32.

Martínez, L.C.A. 2010a. Monocotiledóneas en Auca Mahuevo, Formación Anacleto (Campaniano), Neuquén, Argentina. 10 Congreso Argentino de Paleontologia y Bioestratigrafía y $7^{\circ}$ Congreso Latinoamericano de Paleontología (La Plata). Resúmenes: 182-183.

Martínez, L.C.A. 2010b. [Estudios Xilológicos en el Cretácico de la Cuenca Neuquina, provincia del Neuquén, Argentina. Tesis Doctoral. Universidad Nacional de La Plata, 239 p. Inédita.].

Magallón, S. y Castillo, A. 2009. Angiosperm diversification through time. American Journal of Botany 96: 349-365.

Ottone, E.G. 2007. A new palm trunk from the Upper Cretaceous of Argentina. Ameghiniana 44: 719-725.

Pan, A.D., Jacobs, B.F., Dransfield, J. y Baker, W.J. 2006. The fossil history of palms (Arecaceae) in Africa and new records from the Late Oligocene (28-27 Mya) of north-western Ethiopia. Botanical Journal of the Linnean Society 151: 69-81.

Petriella, B. 1972. Estudio de maderas petrificadas del Terciario Inferior del área central de Chubut (Cerro Bororó). Revista del Museo de la Plata (Nueva serie). Sección Paleontología 41: 159-254.

Ramos, V.A. 1981. Descripción Geológica de la Hoja 33c, Los Chihuidos Norte. Provincia del Neuquén. Servicio Geológico Nacional, Boletín 182. 
Romero, E. 1968. Palmoxylon patagonicum n. sp. del Terciario Inferior de la provincia del Chubut, Argentina. Ameghiniana 5: 417-431.

Ruiz-Estrada, E. y Cevallos-Ferriz, S.R.S. 2009. Palmoxylon enochii sp. nov. de la Formación Olmos (Campaniano superior-Maastrichtiano inferior), Coahuila, México. Ameghiniana 46: 577-585.

Schenk, A. 1882. Die von den Gebrüdern Schlagenwelt in Indien gesammelten fossilen Hölzer. Botanischer Jahrbücher fur Systematik, Pflanzengeschichte und Pflanzengeographie 3: 353-358.

Simpson, M.G. 2006. Plant Systematics. Elsevier Academic Press, Nueva York, 590 p.

Stenzel, G. 1904. Fossile Palmenhölzer. En: V. Uhlig y G. von Arthaber (Eds.), Beiträge Zur Paläontologie und Geologie Österreich-Ungarns und Des Orients. Wienund Leipzig: K.U.K. Hof-und Universitäts-Buchhändler, p. 107-208.

Tomlinson, P.B. 1961. Palmae. En: CR. Metcalfe (Ed.). Anatomy of the Monocotyledons II. Palmae. Oxford University Press. Oxford, 453 p.
Tomlinson, P.B. 2006. The uniqueness of palms. Botanical Journal of the Linnean Society 151: 5-14.

Torres, T.G. y Godoy, E. 1982. Hallazgo de Palmoxylon chilensis n. sp., del Cretácico Superior en Huechun, Región Metropolitana, $3^{\circ}$ Congres Geológico Chileno, Actas: 302-320.

doi: 10.5710/AMGH.7.2.2012.514

Recibido: 1 de julio de 2011

Aceptado: 7 de febrero de 2012 